\title{
One-way translational magnetic mass damper model for structural response control against dynamic loadings
}

\author{
Nurulashikin Bahaman ${ }^{1, *}$, Sk Muiz Sk Abdul Razak ${ }^{1}$, Azlan Adnan ${ }^{2}$, Norrazman Zaiha \\ Zainol $^{1}$, Norhaizura Yahya ${ }^{1}$, Khairunnisa Muhamad ${ }^{1}$, Ahmad Nurfaidhi Rizalman ${ }^{3}$, \\ Mazizah Ezdiani Mohamad ${ }^{4}$, and Nur Adibah Ayuni Abd Malek ${ }^{1}$ \\ ${ }^{1}$ Civil Engineering Technology Department, Faculty of Civil Engineering Technology, Universiti \\ Malaysia Perlis (UniMAP), 02100 Sg. Chuchuh, Padang Besar, Perlis, Malaysia. \\ ${ }^{2}$ Engineering Seismology \& Earthquake Engineering Reaserch (e-SEER), Institute of Noise \& \\ Vibration, Universiti Teknologi Malaysia, UTM, 81310 Skudai, Johor, malaysia. \\ ${ }^{3}$ Civil Engineering Programme, Faculty of Engineering, UMS, 88899 Kota Kinabalu, Sabah, \\ Malaysia. \\ ${ }^{4}$ Department of Civil Engineering, School of Engineering \& Technology, University College of \\ Technology Sibu, 96000 Sibu, Sarawak, Malaysia.
}

\begin{abstract}
Structural responses should be reduced to minimize the consequent structural damage caused by dynamic excitation. The one-way translational magnetic mass damper model is developed as a new type of damper for the purpose of structural response control. The damper utilizes the concept of repulsive force between magnets with same poles to create a magnetic force to stabilize or bring the structure back to its original position. The dynamic performance of the structure was tested using a harmonic shaking table. In this study, the three parameters used are excitation speeds: $2.5 \mathrm{~V}$ (low), $6.0 \mathrm{~V}$ (medium) and $8.5 \mathrm{~V}$ (high); strength of magnets: weak (N35), medium (N45) and strong (N52); and the mass in the damper: $40 \mathrm{~g}$, $101 \mathrm{~g}$ and $162 \mathrm{~g}$. The correlations of the parameters towards the structural displacement are verified in the testing. The displacement is highly reduced up to $100 \%$ at the first level and $85.2 \%$ at the fifth level. The most optimum structural response control was attained when a strong magnetic strength and mass of $162 \mathrm{~g}$ are used. When tested with three excitation speeds; $2.5 \mathrm{~V}, 6.0 \mathrm{~V}$ and $8.5 \mathrm{~V}$, the damper with this setting provides the optimum damping effect towards the structure in terms of displacement.
\end{abstract}

\section{Introduction}

Dynamic loading is an external force exerted in certain amounts on a structure upholding them. The occurrence of dynamic loading or synonymously known as dynamic excitation

* Corresponding author: nurulashikinbahaman@gmail.com 
such as earthquake ground motion, typhoon and machinery vibration may cause severe structural damages to a structure such as building or infrastructure. Earthquake can be is defined as any trembling of the earth's surface which then generates a sudden dislocation of a segment of crust or by a volcanic eruption, or at times by man-made explosions, and is followed by a release of energy in the earth's crust [1].

The occupants of the building may be at risk due to the effect of dynamic excitation and the damage of the structure may put one's life in danger. The exposure of structure towards standard gravity loads and additional dynamic loads created by the tragedy of earthquakes, wind or other natural or technological causes gives a major effect towards the safety of the structure [2].

Therefore, various structural response control devices were proposed by several researchers. The device includes bracing and outrigger systems, damper systems and base isolation systems. Few passive damping systems are viscous dampers, hydraulic dampers and mass dampers.

Viscous dampers are developed since they can be connected in many ways such as installation in the floor or foundation, connecting dampers in stern pericardial braces and in diagonal braces [3]. However, it is has been found that the damper can only work well within the designed working temperature. One of the parameters of the fluid viscous damper is the temperature. The dampers use the increasing temperature of the damping medium to store energy temporarily in order for the dampers to protect the structure from damage. The temperature law test is carried out to ensure the dampers can work properly based on the design working temperature arranged [4]. In addition, there may be a possible leakage at the fluid seal of the damper that affects the damper's ability to function efficiently [5].

Next, the hydraulic damper is a device designed to absorb and damp shock impulses which functions by the conversion of energy from the excitation into another form of energy such as heat energy to be dissipated. One of the components of the damper is an accumulator which functions to maintain initial pressure and store up oil [6]. The disadvantage of hydraulic dampers is the possibility of leakage that can cause the hydraulic damper to lose its function.

Another type of damper used is the mass damper. The main components of the mass damper are mass, spring and damper [2]. A spring is used to control the motion of the damper in accordance with the structure [7]. The disadvantage of the spring is that the spring stiffness can decrease over time. The constant gravitational force will cause the spring stiffness to decrease over time and then it will affect the overall damping rate and hence may adversely affect the structural response performance of the structure [8].

Therefore, a one-way translational magnetic mass damper model is developed and studied as a new device for structural response control. The concept of repulsive force between magnets with the same pole is used in the study. The structural response is based on three parameters which are excitation speed: $2.5 \mathrm{~V}$ (low), 6.0V (medium) and $8.5 \mathrm{~V}$ (high); strength of magnets: weak (N35), medium (N45) and strong (N52); and the mass in the damper: $40 \mathrm{~g}$, $101 \mathrm{~g}$ and $162 \mathrm{~g}$, are studied. The optimum magnetic mass damper is determined based on these parameters. 


\section{Experimental procedure/methodology}

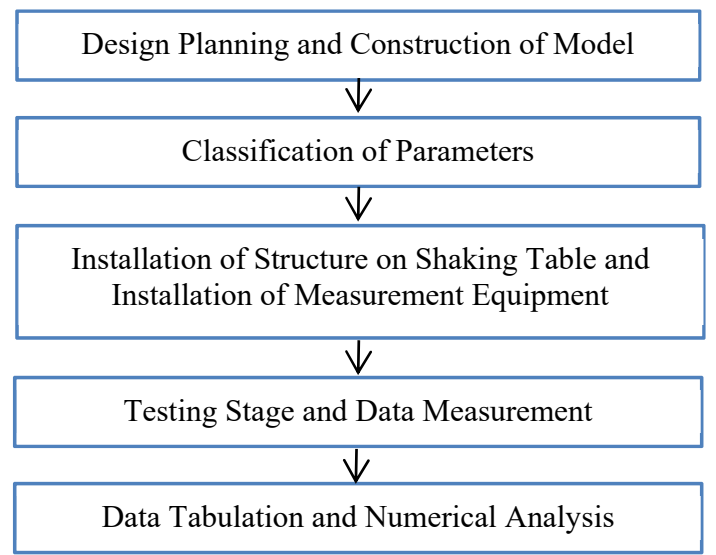

Fig 1. Flowchart of methodology.

\subsection{Design planning and construction of model}

A model is designed according to few criteria. It must be able to withstand the mass exerted on it, additionally the roller is designed so that the model moves in a one-way translational direction. The selected material for the damper is a non-ferromagnetic material in order to avoid magnetic disturbance. The damper is built out of an acrylic and Aluminium material. Meanwhile, the selection of materials for the downscaled model structure is based on aluminium and wood board. Figure 2 and Figure 3 show the design of the structure and magnetic mass damper model. The model is constructed as a 5-storey building.

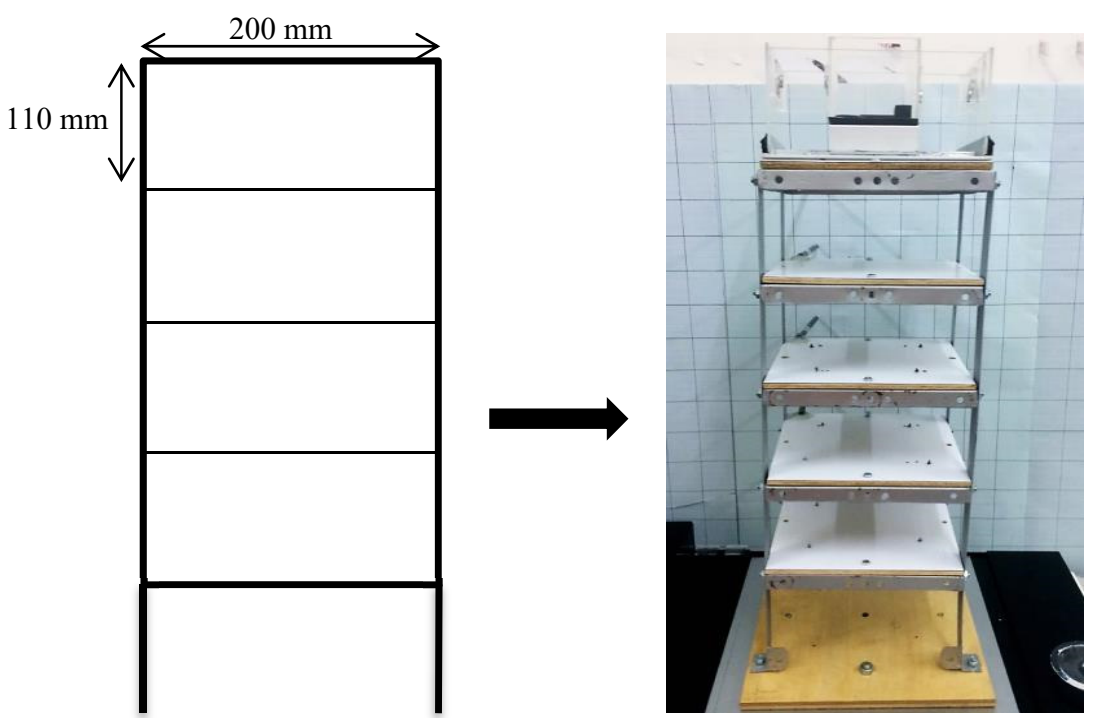

Fig. 2. Five storeys downscaled structure. 


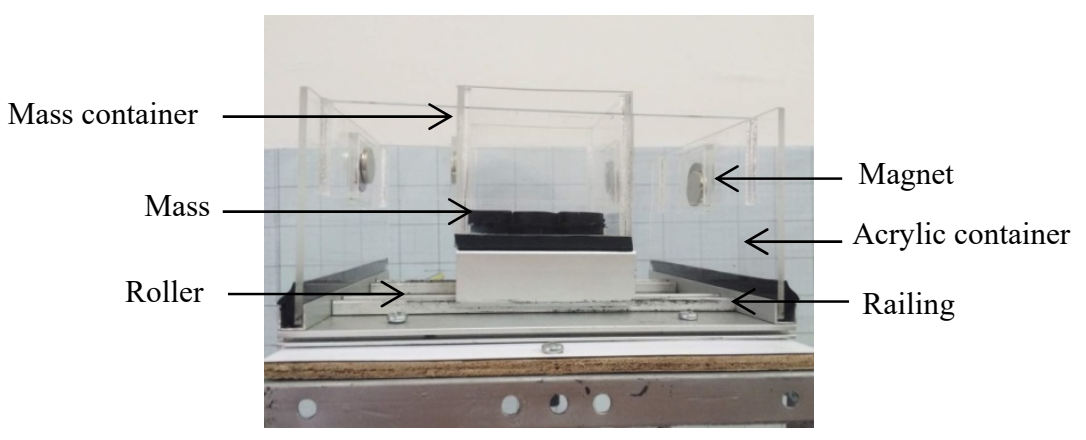

Fig. 3. Design of the damper.

\subsection{Classifications of parameters}

The parameters are the speed of excitation of the shaking table, the magnetic strength of the damper and the mass in the damper. The speeds of excitation used are according to the correlation of Instrumental Intensity with Mercalli Scale. Drift limitation is studied in order to analyse the displacement of the structure subjected to seismic excitation. The drift limitation varies from $0.06 \%$ to $1.0 \%$. Meanwhile, research on the magnet's properties and characteristics is done to analyse the different strengths of permanent magnets.

\subsubsection{Determination of excitation speed of shaking table}

The excitation of the shaking table is determined from the Mercalli scale of earthquake. In correlation with the Mercalli scale, the United States of Geological Survey (USGS) developed a scale called Instrumental Intensity scale. This scale maps the peak ground acceleration and peak ground velocity on an intensity scale that is similar to the one felt in the Mercalli scale. Table 1 shows the specified excitations of the shaking table used in the study.

Table 1. Details of shaking table speed.

\begin{tabular}{|c|c|c|c|}
\hline $\begin{array}{c}\text { Velocity according to } \\
\text { Mercalli scale } \\
(\mathbf{m} / \mathbf{s})\end{array}$ & $\begin{array}{c}\text { Shaking Table } \\
\text { Excitation } \\
(\mathbf{V})\end{array}$ & $\begin{array}{c}\text { Shaking Table } \\
\text { Excitation } \\
(\mathbf{m} / \mathbf{s})\end{array}$ & Perceived shaking \\
\hline $0.011-0.034$ & 2.5 & 0.022 & Light \\
\hline $0.034-0.081$ & 6.0 & 0.040 & Moderate \\
\hline $0.081-0.16$ & 8.5 & 0.13 & Strong \\
\hline
\end{tabular}

\subsubsection{Determination of magnetic strength}

Research on the magnet's properties and characteristics is done to analyse the different strength of permanent magnets. According to studies, Nd-Fe-B magnets have the best properties with the highest residual flux density, coercive force, and also maximum energy product. Therefore, three different strengths of magnets used in the testing are N35 (weak), N45 (medium) and N52 (strong).

\subsubsection{Determination of required mass for damping}

The required mass for damping, which is approximately $5 \%$ of the mass of the structure, is determined. The percentage is taken considering the mass ratio applied by other researchers. Based on the results presented in the study of seismic performance of tune mass dampers, the 
mass ratios between $2 \%$ and $8 \%$ is an appropriate and optimum measure as a control of structural response subjected to seismic ground motions [9]. Mass of the structure is determined since the mass in the damper is dependent on the mass of the structure. The mass of the structure is exactly $2.02 \mathrm{~kg}$ or $2020 \mathrm{~g}$ after weighing. Thus, the three masses are shown in Table 2.

Table 2. Detail of mass used in damper.

\begin{tabular}{|c|c|c|}
\hline \multicolumn{3}{|c|}{ Mass of structure: 2020g } \\
\hline Mass in Damper & Per cent mass ratio (\%) & Mass used (g) \\
\hline M1 & 2 & 40 \\
\hline M2 & 5 & 101 \\
\hline M3 & 8 & 162 \\
\hline
\end{tabular}

\subsection{Installation of structure on shaking table and installation of measurement equipment}

The structure is installed on the shaking table. The base of the structure will be installed on the shaking table while other connections are installed as pin support. The fixed pin is used at the base to ensure stability and to avoid the active movement of structure at the base. The equipment or instruments used to obtain data are an oscilloscope and a vibration meter

\subsection{Testing stage and data measurement}

The finished model is placed on the structure to be tested. A bolt and nut is used as the fixed pin of the model on the slab of the structure. The damper will be placed at the top-most level during testing. Figure 4 shows the damper and structure ready to be tested on the shaking table.

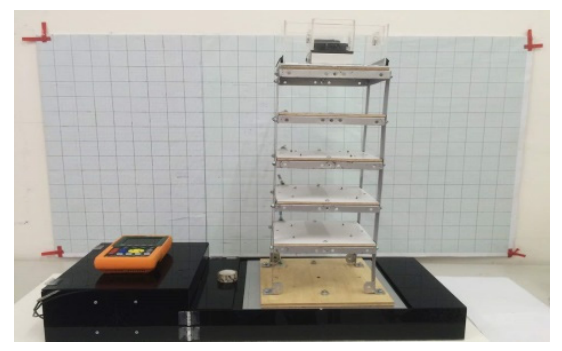

Fig. 4. Damper and structure to be tested on shaking table.

The testing stage will be conducted on the fourth level. A weak magnet is placed on the fourth level and three different masses are used during testing. With each mass in the damper, different speeds of excitation (three levels of excitation) will be excited from the shaking table towards the structure. The testing is repeated three times in order to obtain accurate results. The testing stage is repeated using different strengths of magnets and changing the mass accordingly. The data of the structural displacement is recorded.

\subsection{Data tabulation and numerical analysis}

The results obtained from the testing is recorded. The data is tabulated and a numerical analysis is conducted. The most suitable mass and magnetic strength is determined. 


\section{Results and discussion}

Comparisons between the three specified parameters towards structural displacement are done. The three parameters are excitation speed, strength of magnet and masses in the damper. The damper with a strong magnetic strength with mass of $162 \mathrm{~g}$ is determined to be the most optimum damper. Thus, the reduction in displacement for strong magnetic strength with a mass of $162 \mathrm{~g}$ is shown in the plotted graphs.

\subsection{Magnetic strength influence towards structural response performance}

The graph in Figure 5 shows the structural displacement when high excitation is applied towards magnets with mass of $162 \mathrm{~g}$.

$\leadsto$ WITHOUT DAMPER MAGNET

$\rightarrow$ MEDIUM MAGNET

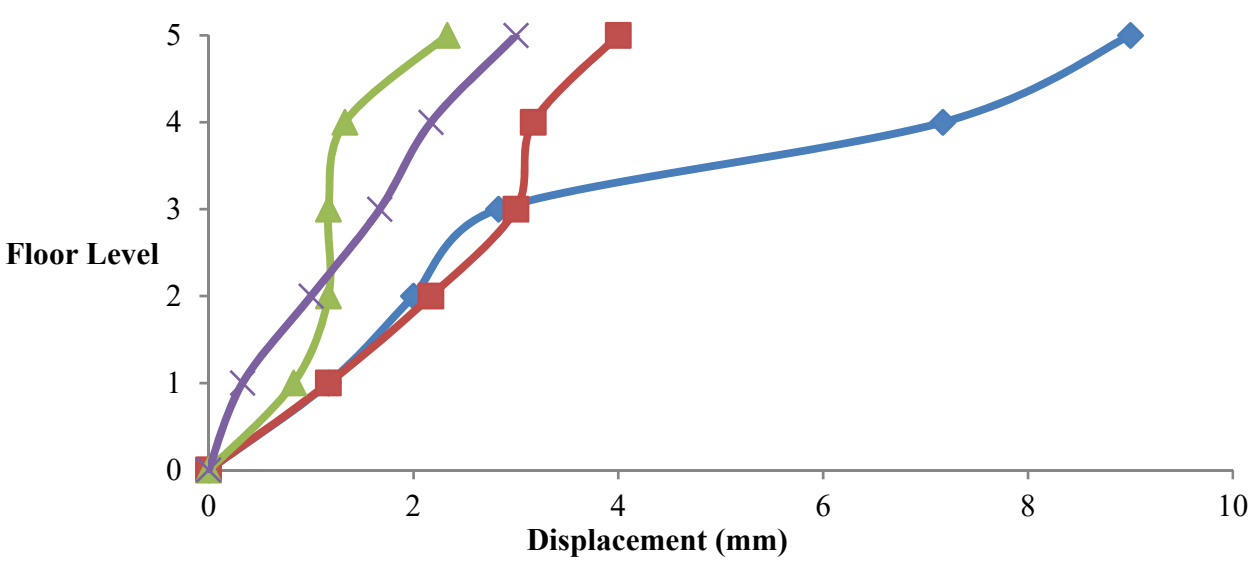

Fig. 5. Strength of magnet vs displacement (high speed, $162 \mathrm{~g}$ mass).

Based on the graph, without a damper, the structural displacement is the highest from the third to the fifth level which is $2.83 \mathrm{~mm}$ to $9.0 \mathrm{~mm}$ while the damper with a weak magnet causes the highest displacement from the first to the second level of the structure. This indicates that the installation of the weak magnet on the damper is ineffective. During the motion of structure and damper, the weak strength of the magnet is unable to provide an adequate repulsive force to bring the damper and structure back to the original position.

Next, the damper containing medium and strong magnets greatly reduced the structural displacement when compared to the structural displacement without a damper. The graph pattern shows that the reduction of structural displacement is the highest when using medium and strong magnetic dampers. The minimum and the maximum displacements recorded when using a strong magnetic damper is $0.33 \mathrm{~mm}$ and $3.0 \mathrm{~mm}$ respectively, while the minimum and the maximum displacements when using a medium magnetic damper is $0.83 \mathrm{~mm}$ and $2.33 \mathrm{~mm}$ respectively. A slight difference is seen at the first level where the reduction of displacement for the medium magnet is a little lower than that for a damper with strong magnet. However, the damper with a medium magnet causes the highest reduction of structural displacement at the upper level. Thus, when applying a mass of 162g and high excitation speed, the damper with a medium magnet is most effective towards the upper level while the damper with a strong magnet is most effective towards the lower level.

Thus, when applying a mass of $162 \mathrm{~g}$ and a high excitation speed $(8.5 \mathrm{~V})$, the damper with a strong magnet is most effective. 


\subsection{Mass influence towards structural response performance}

The graph in Figure 6 shows the structural displacement when high excitation is applied towards a strong magnet with mass of $40 \mathrm{~g}, 101 \mathrm{~g}$ and $162 \mathrm{~g}$.

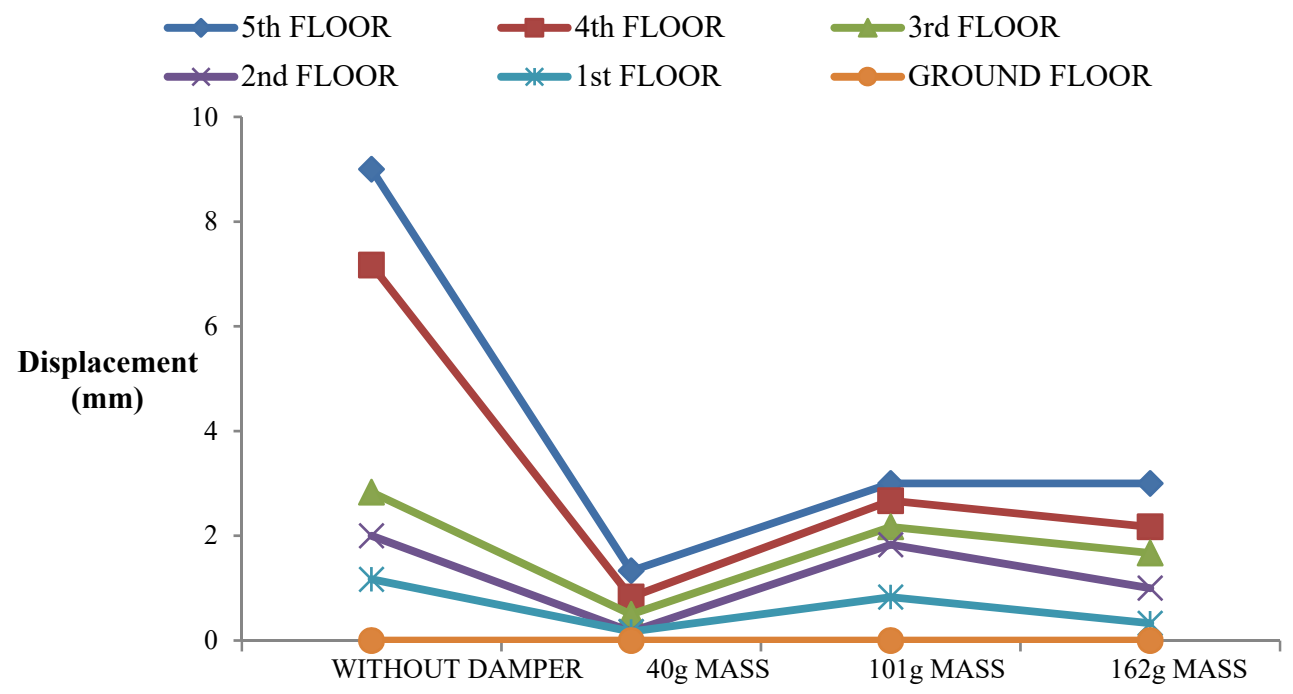

Fig. 6. Mass vs Displacement (High Speed, Strong Magnet).

From the graph, without a damper, mode shape of the structure is the most critical where the displacements vary greatly from $1.17 \mathrm{~mm}$ at the bottom to $9.0 \mathrm{~mm}$ at the top level. When the damper containing a mass of $40 \mathrm{~g}$ is installed, the structural displacement is greatly reduced at each level. It also gave the highest reduction in structural displacement when compared to the other damper. The minimum displacement when using $40 \mathrm{~g}$ masses is $0.17 \mathrm{~mm}$ while $0.83 \mathrm{~mm}$ and $0.33 \mathrm{~m}$ when using $101 \mathrm{~g}$ and $162 \mathrm{~g}$ masses respectively. Meanwhile, the maximum displacement is at the top most level of the structure which is $1.33 \mathrm{~mm}, 3.0 \mathrm{~mm}$ and $3.0 \mathrm{~mm}$ respectively. During the motion of structure and damper, while the strong strength of the magnet provides an adequate repulsive force to bring the damper and structure back to the original position, the mass in the damper functioned to dissipate the high energy of excitation $(8.5 \mathrm{~V})$. This causes the reduction of structural displacement.

However, the displacement rises up to 0.83 and $0.33 \mathrm{~mm}$ for masses $101 \mathrm{~g}$ and $162 \mathrm{~g}$. The $101 \mathrm{~g}$ and $162 \mathrm{~g}$ mass in the damper is unable to dissipate the energy of excitation but added the load towards the structure and causes deflection instead. The mass is considered excessive and makes it impossible for the structure to withstand the mass while experiencing excitation from the shaking table. This indicates that the damper containing a mass of $40 \mathrm{~g}$ is the most effective to reduce the structural displacement while the dampers containing masses of $101 \mathrm{~g}$ and $162 \mathrm{~g}$ are considered less effective and uneconomical.

Overall, for strong magnetic damper, a mass of $40 \mathrm{~g}$ is the most effective to reduce the structural displacement when a high speed of excitation $(8.5 \mathrm{~V})$ is applied.

\section{Conclusion}

The most optimum damper is concluded based on each specified parameter. As a conclusion, the most optimum magnetic mass damper is one that uses the strong magnetic strength containing a mass of $162 \mathrm{~g}$. When tested with three speeds of excitation; $2.5 \mathrm{~V} 6.0 \mathrm{~V}$ and $8.5 \mathrm{~V}$, the damper provided the most optimum damping effect towards the structural displacement. 
The testing shows that the displacement is highly reduced up to $100 \%$ at the first level and $85.2 \%$ at the fifth level. The one-way magnetic mass damper contributed to a few aspects environmentally, economically and safety. A one-way translational magnetic mass damper uses the forces from the magnets to provide a damping effect towards structure. The damper does not require or use oil fuel or electricity to function. The wastage of oil and fuel may cause pollution to the environment. The magnetic mass damper requires less maintenance rather than the damper that uses a spring or a hydraulic as a spring may reduce its stiffness over time and the hydraulic damper has a possibility to leak. In contrast, a magnet is not subjected to stiffness or leakage. Therefore, the magnetic mass damper is more economical because it does not require frequent maintenance and hence the maintenance cost will be lower.

The author would like to acknowledge the support from the Fundamental Research Grant Scheme (FRGS) under a grant number of FRGS/1/2017/SS03/UNIMAP/03/1 from the Ministry of Higher Education Malaysia.

\section{References}

1. S. Jain, Earthquake. Fundamentals of Physical Geology, 338-369 (2014)

2. Z. T. Rakicevic, A. Bogdanovic, D. Jurukovski, \& P. Nawrotzki, Effectiveness of tune mass damper in the reduction of the seismic response of the structure (Bull Earthquake Engineering). 1049-1073 (2012)

3. A. Heysami, Type of Dampers and their Seismic Performance During an Earthquake. 1002-1015. (2015)

4. F. Qian, S. Ding \& J. Song, Testing of Fluid Viscous Damper. China: Shanghai Research Institute of Materials. (2012)

5. P. Castaldo, Passive Energy Dissipation Devices. In Integrated Seismic Design of Structure and Control Systems, 21-62 (2012)

6. M. H. Shih \& W. P. Sung, Development of semi-active hydraulic damper as active interaction control device to withstand external excitation, 123-138 (2014)

7. V. Matsagar, Advances in Structural Engineering, Dynamic. 2, (2015)

8. S. M. Razak, A. Adnan, M. Z. Ramli, M. A. Azizan, Z. A. Zahid, M. B. Manaf, et al., Feasibility Assessment of Levitating Magnetic Damper for Structural Response Control. International Journal of Civil Engineering and Geo-Environment. (2015)

9. G. Lavanya \& K. M. Murad, Dyanamic Resistence of Tall Buildings By using Tuned Mass Damper's. International Journal of Advance Engineering and Research Development. (2015) 